\title{
COMPARATIVE MASTICATORY PERFORMANCE IN RESTORED AND NON-RESTORED CHEWING SIDES OF KENNEDY CLASS III SUBJECTS: A CROSS SECTIONAL STUDY
}

\author{
Neveen S. Abd El Rahim ${ }^{* * *}$ and Sary Borzangy***
}

\begin{abstract}
Objectives: To assess the masticatory performance of restored short span Kennedy class III edentulous area with respect to non-restored side in patient treated with either fixed dental prostheses (FDPs) and/or removable partial dentures (RPDs).

Methods: Fifty Kennedy class III partially dentate patients with age range (35 - 54) years were recruited for this study. FDPs and/or RPDs were made for all patients. Patients were categorized to five groups; ten patients each. Masticatory efficiency has been evaluated one month after prostheses delivery. Data was analyzed using student's t- test, paired t-test and multi-level linear model test.

Results: Statistical significant difference was observed between different groups under study compared to control group $(\mathrm{p} \leq 0.05)$. However, no statistically significant difference was observed between study groups in respect to age, number of teeth or masticatory efficiency $(p \geq 0.05)$.

Conclusions: Masticatory efficiency at the intact side is better than the side restored with FDPs and /or RPDs. Type of the prosthesis whether FDPs and/or RPDs didn't influence the masticatory efficiency of short span Kennedy class III patients.
\end{abstract}

\section{INTRODUCTION}

The prevalence of tooth loss and the need for prosthetic treatment are high both in Middle East and worldwide and affect the well-being of individuals and the population. Tooth loss has an essential impact on the impairment of chew's ability and this may have consequences over patients 'general health.',
Restoration of missing teeth can be achieved with removable or fixed prostheses to reestablish masticatory and aesthetic functions and to minimize the consequences of tooth loss and edentulism. Although the conventional full coverage fixed dental prosthesis (FDP) preferred by patients to restore missing teeth, it requires the preparation of abutments that results in more tissue loss.

\footnotetext{
* Associate Professor of Removable Prosthodontics, Faculty of Dental Medicine for girls, Al Azhar University.

** Associate Professor of Removable Prosthodontics, College of Dentistry, Taibahu University.

*** Assistant Professor of Substitutive Dental Science, College of Dentistry, Taibahu University.
} 
In contrary, a removable partial denture (RPD) does not poses any of the draw backs accompanied with fixed dental prosthesis as little or no abutment teeth preparation is required. However, the main drawback considered for these type of prostheses is that, RPDs are not 'fixed', and difficulty in adaptation to their use were reported with some patients. ${ }^{3-6}$

Masticatory function comprises the relations between functional and morphologic parts of the teeth, temporomandibular joints (TMJs), and neuromuscular system. Mastication is consisting of two separate procedures: food breakdown into pieces by the action of grinding surface of teeth, followed by the breakdown of the selected parts. ${ }^{7,}$ This procedure involve a given number of teeth, bite force, oral motor function, sensory feedback, food dilution and moistening in saliva, formation of bolus, and swallowing. Additionally, late in life, an important psychosocial role of the mastication process was observed especially when gratifying foods becomes one of the principal delights. ${ }^{9}$

Masticatory efficiency can be evaluated by objective or subjective evaluation. For masticatory performance evaluations, many procedures have been utilized to get objective evaluations. One of them, assessment of distributions of particle size in food bolus in such as almonds, silicone rubber and uncooked carrots. ${ }^{10-13}$ Additionally, shapes of chewed wax cubes and mixtures of color have been utilized. However, most of these described methods are consuming time, complicated and needs bulky, expensive equipment for analysis. ${ }^{14-15}$

A novel technique for analysis of masticatory performance was described by Schimmel et al ${ }^{16}$, at which a material of two different color is masticated for a definite number of chewing strokes, and the result is assessed either by naked eye using a reference scale oropto-electronically.

Though studies the improvement in masticatory performance and satisfaction levels of patients have been assessed with tooth-supported prostheses either fixed or removable, but none assessed comparative evaluation of these two treatment options.

Therefore, this study was designed to compare between fixed dental prostheses and removable partial dentures restoring short span Kennedy's class III partially edentulous patients in respect to their effect on the masticatory efficiency of the patients.

The first null hypothesis tested was; there were no statistical significant difference of masticatory performance between intact side and restored Kennedy class III edentulous area of the same patient. The second null hypothesis tested was; no statistical significant change of masticatory efficiency between FDPs and RPDs restored Kennedy class III edentulous area.

\section{MATERIAL AND METHODS}

Fifty Kennedy class III partially edentulous female patients have been selected from the out-patient clinic, Prosthodontics department, College of Dentistry, Taibahu University. The inclusion and exclusion criteria for selection of patients were; all patients had short span Kennedy class III ridges; with missing upper and/or lower second premolar and first molar; and their ages ranged between 35 and 54 years. They didn't have any debilitating systemic diseases that may disturb normal healing process, oral pathologies, TMJ abnormalities or bone diseases. All patients had normal jaw relationship and adequate inter-arch space. They exhibit a relatively good oral hygiene. Patients of para-functional habits were excluded from this study. A period of at least six months was proceeded since last extraction. Patients had no previous prosthetic experience. Patients have written informed consent approved by the university institutional review board (No.TUCDREC/20180504).They were evaluated through preoperative panoramic radiograph to evaluate the remaining teeth and bone at the edentulous ridge area. Before beginning of treatment, patients obtained comprehensive dental treatment; included 
extraction of hopeless teeth, oral hygiene prophylaxis and periodontal treatment and restoration of carious teeth. Then the patients with short span Kennedy class III partially edentulous either maxillary or mandibular were divided into 5 groups; ten patients' each based on the type of prostheses received. The first group (GI) restored with full ceramic FDPs. The second group (GII) restored with cobalt-chromium RPDs. The third group (GIII) restored with maxillary RPDs and mandibular FDPs. The fourth group (GIV) restored with maxillary and mandibular RPDs. The fifth group (GV) restored with maxillary and mandibular FDPs. Standard clinical and laboratory techniques were followed during construction of cobalt-chromium removable and/or full ceramic fixed partial dentures for all patients. Finished and polished removable and/ or fixed partial dentures were delivered for all the patients, figure (fig.(1)).Evaluation of masticatory efficiency of the patients was carried out one month after delivery of removable and/or fixed partial dentures for all patients.

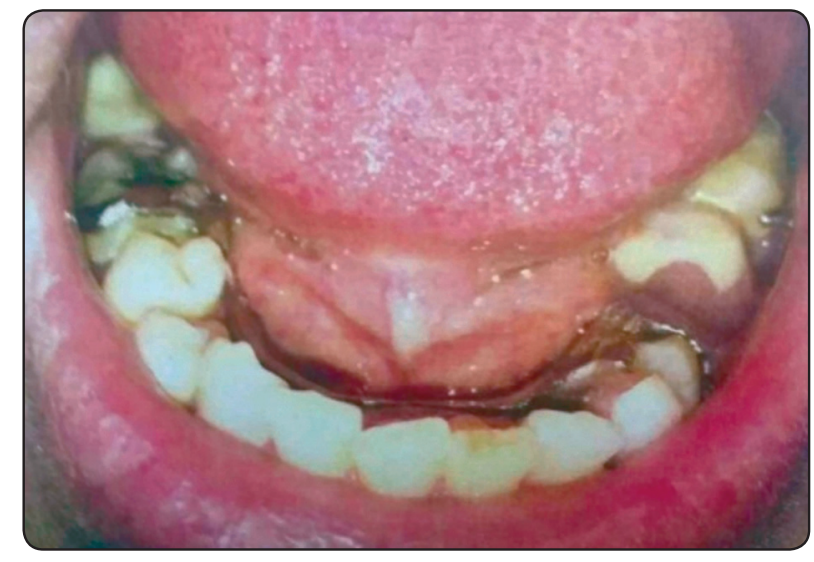

Fig.(1): The finished lower RPD at the patient's mouth.

\section{Masticatory efficiency}

Masticatory efficiency was evaluated after one month from partial denture delivery. Masticatory efficiency test was evaluated using two-colored chewing gums (Lotte ${ }^{\mathrm{TM}}$, Tokyo) comprising a blue and pink beads with the dimensions (19 $\mathrm{mm} \times 14$ $\mathrm{mm} \times 4 \mathrm{~mm})$.

Chewing gum didn't stick to acrylic resin of denture base or artificial teeth and chromium cobalt cast metal frameworks of RPD or to porcelain of FDP. The patient was instructed to sit in upright position and masticate the chewing gum normally 20 times respectively. The patients were instructed to masticate at the intact (non-restored) side with natural maxillary and mandibular teeth opposing each other which is considered the control groups (GI-a, GII-a, GIII- a, GIV-a and GV-a). Then the patient was instructed to masticate at the other side restored with FDPs opposing natural teeth (GIb), RPDs opposing natural teeth (GII-b), FPDs opposing RPDs (GIII-b), RPDs opposing RPDs (GIV-b) or FDPs opposing FDPs (GV-b).Chewing process was repeated three times and the mean of the three recordings were considered the masticatory efficiency mean for that patient. Every bolus of gum was taken from oral cavity after 20 strokes and spreaded between two glasses then compressed to 1 $\mathrm{mm}$ wafer to estimate its color.

Digital images for both sides of the samples were taken. Then evaluated with a new software built program (ViewGum), available for free, (www.dhal.com).The images were transformed into HSI color space. The hue value was calculated per pixel in segmented images. If colors of the images are not assorted, two divided peaks on the hue axis are distinguished. With increasing percentage of color blending, the two hue peaks of different colors approach each other and will unit at an average area as one peak when the colors are completely blended. Therefore, improperly blending presents with more variance on the hue axis than well blending. The variance of the hue $(\mathrm{VOH})$ is evaluated as the estimation of blending; Wallace et a ${ }^{17}$. The images were analyzed randomly by two different operators.

Statistical analysis was performed with Statistical Package for Scientific Studies (SPSS16.0) for 
Windows and using student's t- test, paired t-test, and multi-level linear model test. The significance level was set at $\mathrm{P} \leq 0.05$.

\section{RESULTS}

Fifty Kennedy class III partially edentulous female patients (age $45 \pm 5.6$ years) participated in the study. One month after delivery of RPDs and/ or FPDs for all patients, there were no statistically significant differences observed between the five patient groups; with regards to age $(p=0.808)$ or number of teeth $(p=0.794)$.

- There were statistically significant differences in masticatory efficiency between control non-restored sides (GI-a, GII-a, GIII- a, GIV-a and GVa) and restored sides (GI-b, GII-b, GIII- b, GIV-b and GV-b) respectively, table (1).

- There were no statistically significant differences in mean difference of masticatory efficiency; between non-restored sides and restored sides in each group; of different groups (GI, GII, GIII, GIV and GV) ( $p=0.390)$, fig. (2).

TABLE (1): Paired samples statistics for masticatory efficiency.

\begin{tabular}{|c|c|c|c|c|}
\hline & Mean & $\begin{array}{c}\text { Std. } \\
\text { Deviation }\end{array}$ & $\begin{array}{c}\text { Std. Error } \\
\text { Mean }\end{array}$ & $\begin{array}{c}\text { P } \\
\text { value }\end{array}$ \\
\cline { 2 - 5 } GI-a & 0.158 & \pm 0.0739 & 0.0234 & $0.005^{*}$ \\
\hline $\begin{array}{l}\text { GII-a } \\
\text { GII-b }\end{array}$ & 0.087 & \pm 0.0464 & 0.0147 & $0.005^{*}$ \\
\hline $\begin{array}{c}\text { GIII-a } \\
\text { GIII-b }\end{array}$ & 0.093 & \pm 0.0383 & 0.0121 & $0.012^{*}$ \\
\hline $\begin{array}{l}\text { GIV-a } \\
\text { GIV-b }\end{array}$ & 0.089 & \pm 0.026 & 0.0823 & $0.001^{*}$ \\
\hline $\begin{array}{l}\text { GV-a } \\
\text { GV-b }\end{array}$ & 0.179 & \pm 0.0517 & 0.0164 & $0.013^{*}$ \\
\hline
\end{tabular}

* $P \leq 0.05$, statistically significant.

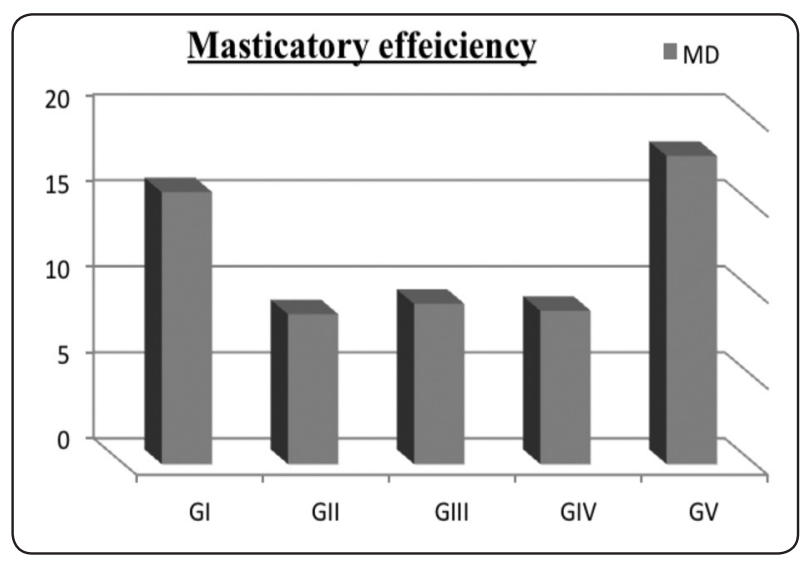

Fig. (2): Mean difference (MD) values of masticatory efficiency between different groups.

\section{DISCUSSIONS}

Improvement of masticatory function of partially dentate patients is an important goal of dental prostheses either fixed or removable. Favorable masticatory performance and occlusal loading that does occur as a result of the stabilization of a proper occlusal relationship have an important impact form physiological point of view. Therefore; routinely attention is paid in prosthetic treatment to the variation of the occlusal morphology design of dental prostheses in order to improve masticatory performance. ${ }^{18,19}$

In this study, in order to measure masticatory efficiency, a two different color material which is chewed for a definite number of cycles and the results that is evaluated by naked eye. From the clinical prospective point of view, the results show that this procedure is an effective tool to evaluate masticatory efficiency from a kinematic point of view, effectively permitting estimation of kinematic changes in the occlusal rehabilitation course. ${ }^{20}$

Mean values from 20 cycles appear adequate for analysis in this study. Mandibular cycles from 10 to cycle 20 on average are favorable for mandibular movements' assessment. Additionally, the chewing material is consistent in composition and permits 
stable masticatory mandibular movements, however, at swallowing; the path of movement becomes unstable. $^{21,22}$

To the best authors' knowledge, no studies have compared conventional FDPs and RPDs modalities in missing teeth replacement with regard to masticatory efficiency and masticatory performance.

This study aimed at comparing the masticatory efficiency of conventional metal framework removable partial dentures and fixed dental prostheses restoring short span Kennedy's class III partially edentulous patients. The result showed that, the masticatory efficiency for partially dentate patient restored with RPDs is better compared to patient restored with FDPs while the difference was non-significant. This could be attributed to the ability of plastic teeth of removable partial denture to simulate the occlusal anatomy of the natural teeth, while for missed teeth restored with FPDs, the ability to stimulate the occlusal anatomy of natural teeth depends up the skills of dental technician which is varies.

Regardless the type of restoration used, the masticatory efficiency of the intact non-restored side showed significant masticatory efficiency compared with restored side. The masticatory efficiency was studied in dentate patients compared with aged dentures wearer; they showed that dentate individuals had significantly more efficiency than did aged denture wearers. ${ }^{23}$

Although, most general practitioners think, from their clinical practices, that the occlusal anatomy of posterior teeth could be linked to the masticatory performance and stability, little or no records regarding to the clinical evidence demonstrating the impact of posterior fixed dental prostheses, such as proximal contacts, occlusal contacts and deflecting contours on the influence on the masticatory cycle..$^{24-26}$

\section{CONCLUSION}

- Masticatory efficiency at the intact side is better than the side restored with FDPs and /or RPDs.

- Masticatory efficiency of short span Kennedy class III patients isn't influenced by type of the prosthesis whether FPDs and/or RPD.

\section{References}

1. Ali Z, Baker SR, Shahrbaf S, Martin N, Vettore MV. Oral health-related quality of life after prosthodontic treatment for patients with partial edentulism: A systematic review and meta-analysis. J Prosthet Dent. 2019 Jan;121(1):59-68.e3.

2. Peeran SA, Al Sanabani F, Al-Makramani BM, Elamin EI. Dental prosthetic status and treatment needs of adult population in Jizan, Saudi Arabia: A survey report. Eur J Dent. 2016 Oct-Dec; 10(4):459-63.

3. Patel PM, Lynch CD, Sloan AJ, Gilmour AS. Treatment planning for replacing missing teeth in UK general dental practice: current trends. J Oral Rehabil. 2010Jul; 37(7):509-17.

4. Nogawa T, Takayama Y, Ishida K, Yokoyama A. Comparison of Treatment Outcome in Partially Edentulous Patients with Implant-Supported Fixed Prostheses and Removable Partial Dentures. Int J Oral Maxillofac Implants. 2016Nov/ Dec;31(6):1376-1383

5. Al-Quran FA, Al-Ghalayini RF, Al-Zu'bi BN. Single-tooth replacement: factors affecting different prosthetic treatment modalities. BMC Oral Health. 2011 Dec21; 11:34-40.

6. Douglass CW, Watson AJ. Future needs for fixed and removable partial dentures in the United States. J Prosthet Dent. 2002 Jan; 87(1):9-14.

7. Andrade KM, Alfenas BFM, Rodrigues Garcia RCM. Influence of removable prostheses on mastication in elderly subjects with rheumatoid arthritis. J Oral Rehabil. 2018 Apr; 45(4):295-300

8. Radi IA, Taha K. No clear evidence on the clinical performance of different removable prosthetic options in partially edentulous patients. Evid Based Dent.2017 Dec 22; 18(4):117-18.

9. Ishida K, Nogawa T, Takayama Y, Saito M, Yokoyama A. Prognosis of double crown-retained removable dental prostheses compared with clasp-retained removable dental prostheses: A retrospective study. J Prosthodont Res. 2017 Jul; 61(3):268-75. 
10. Schimmel M, Memedi K, Parga T, Katsoulis J, Müller F. Masticatory Performance and Maximum Bite and Lip Force Depend on the Type of Prosthesis. Int J Prosthodont. 2017 November/December; 30(6):565-72.

11. Silva LC, Nogueira TE, Rios LF, Schimmel M, Leles CR. Reliability of a two-colour chewing gum test to assess masticatory performance in complete denture wearers. J Oral Rehabil. 2018 Apr; 45(4):301-307.

12. Halazonetis DJ, Schimmel M, Antonarakis GS, Christou P. Novel software for quantitative evaluation and graphical representation of masticatory efficiency. J Oral Rehabil. 2013 May; 40(5):329-35.

13. Vaccaro G, Pelaez JI, Gil JA. Choosing the best image processing method for masticatory performance assessment when using two-coloured specimens. J Oral Rehabil. 2016 Jul; 43(7):496-504.

14. Müller F, Hernandez M, Grütter L, Aracil-Kessler L, Weingart D, Schimmel M. Masseter muscle thickness, chewing efficiency and bite force in edentulous patients with fixed and removable implant-supported prostheses: a cross-sectional multicenter study. Clin Oral Implants Res. 2012 Feb; 23(2):144-50.

15. Weijenberg RA, Scherder EJ, Visscher CM, Gorissen T, Yoshida E, Lobbezoo F. Two-colour chewing gum mixing ability: digitalisation and spatial heterogeneity analysis. J Oral Rehabil. 2013 Oct; 40(10):737-43.

16. Schimmel M, Christou P, Miyazaki H, Halazonetis D, Herrmann FR, Müller F. A novel colourimetric technique to assess chewing function using two-coloured specimens: Validation and application. J Dent. 2015 Aug;43(8):955-64.

17. Wallace S, Samietz S, Abbas M, McKenna G, Woodside JV, Schimmel M. Impact of prosthodontic rehabilitation onthe masticatory performance of partiallydentate older patients: Can it predict nutritional state? Results from a RCT.J Dent. 2018 Jan; 68:66-71.
18. Bourdiol P, Mioche L. Correlations between functional and occlusal tooth-surface areas and food texture during natural chewing sequences in humans. Arch Oral Biol. 2000 Aug; 45(8):691-9.

19. Müller F, Shimazaki Y, Kahabuka F, Schimmel M.Oral health for an ageing population: the importance of a natural dentition in older adults. Int Dent J. 2017 Sep; 67 Suppl 2:7-13.

20. Tarkowska A, Katzer L, Ahlers MO. Assessment of masticatory performance by means of a color-changeable chewing gum. J Prosthodont Res. 2017 Jan; 61(1):9-19.

21. Komagamine Y, Kanazawa M, Minakuchi S, Uchida T, Sasaki Y. Association between masticatory performance using a colour-changeable chewing gum and jaw movement. J Oral Rehabil. 2011 Aug; 38(8):555-63.

22. Uesugi H, Shiga H. Relationship between masticatory performance using a gummy jelly and masticatory movement. J Prosthodont Res. 2017 Oct; 61(4):419-425.

23. Mishellany-Dutour A, Renaud J, Peyron MA, Rimek F, Woda A.Is the goal of mastication reached in young dentates, aged dentates and aged denture wearers? Br J Nutr. 2008 Jan; 99(1):121-8.

24. Watamoto T, Egusa H, Mizumori T, Yashiro K, Takada K, Yatani H. Restoration of occlusal and proximal contacts by a single molar crown improves the smoothness of the masticatory movement. J Dent. 2008 Dec; 36(12):984-92.

25. Watamoto T, Mizumori T, Egusa H, Yashiro K, Takada K, Yatani $\mathrm{H}$. The influence of single molar crown placement on the smoothness of masticatory movement. J Oral Rehabil. 2008 Jun; 35(6):440-5.

26. Jepson N, Allen F, Moynihan P, Kelly P, Thomason M.Patient satisfaction following restoration of shortened mandibular dental arches in a randomized controlled trial. Int J Prosthodont. 2003 Jul-Aug; 16(4):409-14. 